\title{
Antiaromaticity in Bare Deltahedral Silicon Clusters Satisfying Wade's and Hirsch's Rules: An Apparent Correlation of Antiaromaticity with High Symmetry
}

R. Bruce King, ${ }^{* a}$ Thomas Heine, ${ }^{b}$ Clémence Corminboeuf ${ }^{c}$ and Paul v. R. Schleyer* ${ }^{* a}$

${ }^{a}$ Department of Chemistry and Center for Computational Quantum Chemistry, University of Georgia, Athens, Georgia 30602 USA; Institut für Physikalische Chemie und Elektrochemie, Technische Universität Dresden, D-01062 Dresden, Germany;

Department of Physical Chemistry, University of Geneva, 30 quai Ernest-Ansermet, CH-1211, Genève 4, Switzerland

\section{SUPPORTING INFORMATION}

\section{Gaussian Archive Entries: $\mathrm{Si}_{n}{ }^{2-}$ Minima at B3LYP/6-311+G*}

\begin{abstract}
$1 \backslash 1 \backslash F A U-C C C-D E R I O C 3 \backslash F R E Q \backslash R B 3 L Y P \backslash 6-311+G$ (D) \SI5 (2-) \SCHLEYER $\backslash 29-J u 1-1996 \backslash 0 \backslash \backslash \#$ BE

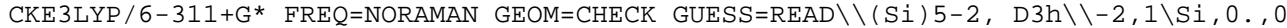
$, 1.8710761837 \backslash \mathrm{Si}, 0,1.5044416678,0 . \backslash \mathrm{Si}, 1.3028847029,-0.7522208339,0 . \backslash \mathrm{Si},-1.302$ $8847029,-0.7522208339,0 . \backslash \mathrm{Si}, 0 ., 0 .,-1.8710761837 \backslash \backslash$ Version=HP-PARisC-HPUX-G94RevC $.3 \backslash$ Stat $e=1-A 1 ' \backslash H F=-1447.524907^{\prime} \backslash \mathrm{RMSD}^{\prime}=2.978 \mathrm{e}-10 \backslash \mathrm{RMSF}=2.833 \mathrm{e}-05 \backslash \mathrm{ZPE}=4.0314833 \backslash \mathrm{Dip}$ ole $=0 ., 0 ., 0 . \backslash$ DipoleDeriv $=-0.3151288,0 ., 0 ., 0 .,-0.3151261,-0.0000122,0 .,-0.000014$ $7,-0.5070103,-0.4872386,0 ., 0 ., 0 .,-0.4259307,0 ., 0 \ldots, 0 .,-0.3286669,-0.4412548,-0.0$ $265531,0 .,-0.0265517,-0.4719101,0.0 ., 0 .,-0.3286616,-0.4412548,0.0265531,0 ., 0.0$ $265517,-0.4719101,0 ., 0 ., 0 .,-0.3286616,-0.3151288,0 ., 0 ., 0 .,-0.3151261,0.0000122$, $0 ., 0.0000148,-0.5070103 \backslash \mathrm{Polar}=276.6166709,0 ., 276.6149913,0 ., 0 ., 383.4248022 \backslash \mathrm{PG}=\mathrm{D}$ $03 \mathrm{H}[\mathrm{C} 3(\mathrm{Si} 1 . \mathrm{Si1}), 3 \mathrm{C} 2$ (Si1)] \NImag=0 \\0.03044615,0.,0.03044591,0.,0.00000006,0.14 $10 ., 0 ., 0.00007710,0 .,-0.00000705,0 .,-0.00000611,0.00000353,0 ., 0.00000611,0.0000$ $0353,0 ., 0 ., 0 .,-0.00007710 \backslash \backslash \backslash €$
\end{abstract}

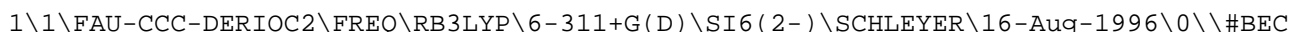

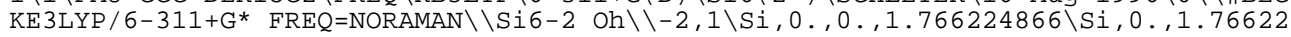
$4866,0 . \backslash \mathrm{Si}, 1.766224866,0 ., 0 . \backslash \mathrm{Si}, 0 .,-1.766224866,0 . \backslash \mathrm{Si},-1.766224866,0 ., 0 . \backslash \mathrm{Si}, 0 .$, $0 .,-1.766224866 \backslash \backslash$ Version=HP-PARisC-HPUX-G94RevC.3 \HF=-1737.055184 \RMSD=6.353e-0 $9 \backslash \mathrm{RMSF}=2.180 \mathrm{e}-05 \backslash \mathrm{ZPE}=5.11632 \backslash \mathrm{Dipole}=0 ., 0 ., 0 . \backslash$ DipoleDeriv $=-0.4638758,0 ., 0 ., 0 .,-0$ $4639173,0,0,0,-0.0720235,-0.4638758,0,0,0,-0.0720235,0 \ldots, 0,0,-0.4639173$ $,-0.0721359,0,0,0,-0.4639883,0,0,0,-0.4639676,-0.4638758,0,0,0,-0.0720$ $235,0 ., 0 ., 0 .,-0.4639173,-0.0721359,0 ., 0 ., 0 .,-0.4639883,0 ., 0 ., 0 .,-0.4639676,-0.4$ $638758,0 ., 0 ., 0 .,-0.4639173,0 ., 0.0 .,-0.0720235 \backslash \operatorname{Pol} 0.0339 .91921,0 ., 339.9457632,0$ $, 0 ., 339.9457621 \backslash \mathrm{PG}=\mathrm{OH} \quad[3 \mathrm{C} 4(\mathrm{Si} 1 . \mathrm{Si} 1)] \backslash \mathrm{NImag}=0 \backslash \backslash 0.04614098,0 \ldots 0.04613509,0 ., 0 ., 0$ $10 \ldots, 0 ., 0.00003763,0 ., 0.00003763,0 ., 0.00003802,0 \ldots, 0 \ldots, 0 \ldots,-0.00003763,0 .,-0.000038$ $02,0 ., 0 ., 0 ., 0 .,-0.00003763 \backslash \backslash \backslash 0$

$1 \backslash 1 \backslash$ FAU-CCC-AW433B $\backslash$ Freq $\backslash$ RB 3LYP \6-311+G (d,p) \Si7 (2-) \SCHLEYER $\backslash 29-N o v-2002 \backslash 0 \backslash \backslash \# R$ $\mathrm{B} 3 \mathrm{LYP} / 6-311+\mathrm{G} * \star \mathrm{SYMM}=\mathrm{LOOSE} \mathrm{POP}=(\mathrm{REG}, \mathrm{NBO}) \quad \mathrm{FREQ}=\mathrm{NORAMAN} \backslash \backslash \mathrm{SI} 7-2 \mathrm{D} 5 \mathrm{H} \backslash \backslash-2,1 \backslash \mathrm{Si}, 0$. $1.665349676,0 . \backslash \mathrm{Si}, 0 ., 1.665349676,0 . \backslash \mathrm{Si},-1.6560714175,0,-1.2032063142 \backslash \mathrm{Si},-1.656$ $0714175,0,1.2032063142 \backslash \mathrm{Si}, 0.6325629937,0,-1.9468287119 \backslash \mathrm{Si}, 0.6325629937,0,1.9$ $468287119 \backslash \mathrm{Si}, 2.0470168476,0.0 . \backslash \backslash$ Version=DEC-AXP-OSF $/ 1-G 98 \mathrm{RevA} .11 .3 \backslash \mathrm{HF}=-2026.56$ $2846 \backslash \mathrm{RMSD}=4.493 \mathrm{e}-09 \backslash \mathrm{RMSF}=2.172 \mathrm{e}-05 \backslash \mathrm{ZPE}=6.0035438 \backslash \mathrm{Dipole}=0.0000794,0 ., 0.0000577 \backslash$ DipoleDeriv $=-0.3160102,0.0001015,0.0000713,-0.0000008,0.1057106,-0.0000006,0.00$ $00713,0.0000737,-0.3160564,-0.31601,-0.0001015,0.0000713,0.0000008,0.1057105,0$. $0000006,0.0000713,-0.0000738,-0.3160565,-0.270175,-0.0000001,0.010466,0 .,-0.442$ $2413,0,0.0104659,0.0000001,-0.2769763,-0.2701984,-0.0000001,-0.0105262,0 .,-0.4$ Polar $=373.5342171,0.0000009,337.516145,-0.0393288,0.0000009,373.5597754 \backslash P G=D 05 \mathrm{H}$ [C5 (Si1.Si1), 5C2 (Si1)] \NImag=0\\0.03848683,-0.00000752,0.08392211,0.00004062,$10.00001699,-0.00004985,0.00001234,0.00001699,0.00004985,0.00001234,-0.00004088$ $\backslash \backslash 0$

$1 \backslash 1 \backslash$ GINC-RAGUE3 $\backslash$ Freg $\backslash$ RB 3LYP $\backslash 6-311+G(d) \backslash$ Si $8(2-) \backslash S C H L E Y E R \backslash 30-N o v-2002 \backslash 0 \backslash \backslash \# N$ GEOM= ALLCHECK GUESS=TCHECK RB3LYP /6-311+G(D) FREQ \\Si8-2 D2dTS->D2 \-2, $1 \backslash S i, 0.016681$ $1904,1.8472408792,0.4340138929 \backslash \mathrm{Si},-0.0166811904,-1.8472408792,0.4340138929 \backslash \mathrm{Si}, 1$ $.8472408792,0.0166811904,-0.4340138929 \backslash \mathrm{Si},-1.8472408792,-0.0166811904,-0.434013$ $8929 \backslash \mathrm{Si}, 0.1569988281,1.179671037,-1.9031460153 \backslash \mathrm{Si},-0.1569988281,-1.179671037,-1$ $.9031460153 \backslash \mathrm{Si}, 1.179671037,0.1569988281,1.9031460153 \backslash \mathrm{Si},-1.179671037,-0.1569988$ $281,1.9031460153 \backslash \backslash$ Version=x86-Linux-G98RevA. 7 $\backslash$ State $=1-A \backslash H F=-2316.0733541 \backslash \mathrm{RMSD}=2$ $.516 \mathrm{e}-09 \backslash \mathrm{RMSF}=3.159 \mathrm{e}-05 \backslash \mathrm{ZPE}=6.5650765 \backslash \mathrm{Dipole}=0 ., 0 ., 0 . \backslash \mathrm{DipoleDer} \mathrm{V} \mathrm{V}=-0.3730328,0$. $022796,-0.0165044,-0.0588711,-0.1708687,-0.0911709,0.0533812,-0.0340509,-0.2541$ $844,-0.3730331,0.0227957,0.0165049,-0.0588706,-0.1708681,0.0911716,-0.0533808,0$ Polar $=369.1142315,11.0184941,369.1140275,-0.0000288,-0.0002825,415.4034769 \backslash P G=D$ $02[\mathrm{X}(\mathrm{Si}) \mathrm{s})] \mathrm{NImag}=0 \backslash \backslash 0.02681132,-0.00712779,0.06557586,0.00194507,0.01343649,0$. $10.00001738,-0.00000469,0.00000479,-0.00001738,0.00000469,0.00000479,-0.0000046$ $\backslash \backslash$ @

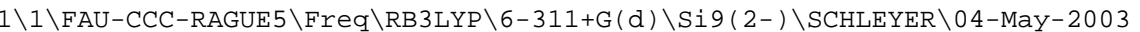

$\backslash 0 \backslash \backslash \# N$ GEOM=ALLCHECK GUESS=TCHECK RB3LYP/6-311+G(D) FREQ $\backslash \backslash S i 9(2-)$ C2v-

>D3h \\-2,1\Si,1.3136130919,0.7736372671,-1.3399790532\Si,-1.3136130919 , $0.7736372671,-1.3399790532 \backslash \mathrm{Si},-1.3136130919,0.7736372671,1.3399790532$

$\backslash \mathrm{Si}, 1.3136130919,0.7736372671,1.3399790532 \backslash \mathrm{Si}, 1.3136130919,-1.54727453$

$41,0 . \backslash \mathrm{Si},-1.3136130919,-1.5472745341,0 . \backslash \mathrm{Si}, 0 .,-1.1985062766,-2.0758737$ 
$643 \backslash \mathrm{Si}, 0 \ldots,-1.1985062766,2.0758737643 \backslash \mathrm{Si}, 0 ., 2.3970125532,0 . \backslash \backslash$ Version $=x 8$ $6-$ Linux-G98RevA.11.3 State $=1-\mathrm{A} 1$ ' $\backslash \mathrm{HF}=-2605.6271482 \backslash \mathrm{RMSD}=2.600 \mathrm{e}-10 \backslash \mathrm{RMSF}=$

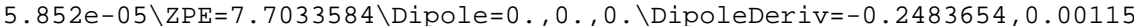
$44,-0.0024423,0.042936,-0.2913519,-0.0201114,-0.0743662,-0.0198944,-0$. $2683359,-0.2483654,-0.0011544,0.0024423,-0.042936,-0.2913519,-0.020111$ $4,0.0743662,-0.0198944,-0.2683359,-0.2483579,-0.0012689,-0.0024192,-0$. $0429577,-0.2913595,0.0200491,-0.0744043,0.0199406,-0.2683121,-0.248357$ $9,0.0014606,0.0023085,0.0429572,-0.29139,0.019923,0.0744046,0.0200315$ $-0.2682816,-0.2483654,-0.0026923,-0.0002214,-0.085871,-0.2567668,0.000$ $0733,0.0000006,-0.0001437,-0.302921,-0.2483654,0.0026923,0.0002214,0.0$ $85871,-0.2567668,0.0000733,-0.0000006,-0.0001437,-0.302921,-0.1700565$, $0 ., 0 ., 0 .,-0.0662752,-0.0706047,0 .,-0.0706047,-0.1478024,-0.1699734,0$. $0 ., 0 .,-0.0662416,0.0705848,0.0 .0706892,-0.1477623,-0.1699734,0 \ldots, 0.0$. $-0.1885555,0.0000713,0 .,-0.0000332,-0.0254483 \backslash$ Polar $=362.7567873,-0.00$ $00009,403.8290903,-0.0000007,0.0207559,403.8530573 \backslash \mathrm{PG}=\mathrm{D} 03 \mathrm{H} \quad[3 \mathrm{C} 2(\mathrm{Si}), 3$ $\mathrm{SGV}(\mathrm{Si} 2)] \backslash \mathrm{NImag}=0 \backslash \backslash 0.05692297,0.00833521,0.05966988,-0.01443738,0.0053$ $2371,0.05352377,-0.03894225,0.00096441,-0.00166980,0.05692297,-0.00096$

$1 \backslash 1 \backslash$ FAU-CCC-RAGUE $4 \backslash$ Freq $\backslash$ RB 3LYP $\backslash 6-311+G(d) \backslash S i 10(2-) \backslash S C H L E Y E R \backslash 29-N o v-2002 \backslash 0 \backslash \backslash \# N$ G EOM=ALLCHECK GUESS=TCHECK RB3LYP/6-311+G(D) FREQ \\Si10-2, D4d \\-2, 1\Si, 0.970962 $2907,0.7345673637,-1.7734326048 \backslash \mathrm{Si}, 0.9709622907,1.7734326048,0.7345673637 \backslash \mathrm{Si}, 0$. $9709622907,-0.7345673637,1.7734326048 \backslash \mathrm{Si}, 0.9709622907,-1.7734326048,-0.73456736$ $37 \backslash \mathrm{Si},-0.9709622907,-0.7345673637,-1.7734326048 \backslash \mathrm{Si},-0.9709622907,1.7734326048,-$ $0.7345673637 \backslash \mathrm{Si},-0.9709622907,0.7345673637,1.7734326048 \backslash \mathrm{Si},-0.9709622907,-1.773$ $4326048,0.7345673637 \backslash \mathrm{Si},-2.5866330654,0 ., 0 . \backslash \mathrm{Si}, 2.5866330654,0 ., 0 . \backslash \backslash$ Version $=x 86-$ Linux-G98RevA.11.3 \State=1-A $\backslash$ HF $=-2895.1740338 \backslash$ RMSD $=6.552 \mathrm{e}-10 \backslash \mathrm{RMSF}=1.100 \mathrm{e}-05 \backslash \mathrm{ZP}$ $\mathrm{E}=9.0969889 \backslash \mathrm{Dipole}=0 ., 0 ., 0 . \backslash \mathrm{DipoleDeriv}=-0.3130165,-0.0134244,0.0325467,-0.0583$ $652,-0.2516299,-0.0171134,0.1412155,-0.0171465,-0.2173942,-0.3130165,-0.0325467$ Polar $=457.2925378,-0.0000001,403.4683067,-0.0000006,0.0000002,403.4683074 \backslash P G=D 0$ $4[\mathrm{C} 4(\mathrm{Si1} . \mathrm{Si1}), \mathrm{X}(\mathrm{Si}) \mathrm{s}] \backslash \mathrm{NImag}=0 \backslash \backslash 0.09076511,0.00485771,0.04802341,-0.01178890,-0$ $\backslash-0.00001441,0.00000300,-0.00000693,-0.00001441,0.00000693,0.00000300,-0.000014$ $\backslash \backslash$ Q

$1 \backslash 1 \backslash$ FAU-CCC-RAGUE $4 \backslash$ Freg $\backslash$ RB 3LYP $\backslash 6-311+G(d) \backslash$ Si11 (2-) \SCHLEYER $\backslash 03-M a y-200$ $3 \backslash 0 \backslash \backslash \# N$ GEOM=ALLCHECK GUESS=TCHECK RB3LYP $/ 6-311+G$ (D) FREO \Si11(2-) C2 $\mathrm{V} \backslash \backslash-2,1 \backslash \mathrm{Si},-0.3237691452,-1.9999820201,1.2414434402 \backslash \mathrm{Si},-0.3237691452,1$ $.9999820201,1.2414434402 \backslash \mathrm{Si},-0.3237691452,1.9999820201,-1.2414434402 \backslash \mathrm{S}$ $i,-0.3237691452,-1.9999820201,-1.2414434402 \backslash \mathrm{Si},-1.511169984,0 ., 2.30992$ $87946 \backslash \mathrm{Si},-1.511169984,0 .,-2.3099287946 \backslash \mathrm{Si}, 1.8369686859,-1.2734711893,0$ . \Si,1.8369686859,1.2734711893,0.\Si,1.1370242295,0.,2.0996947007\Si,1 $.1370242295,0 \ldots,-2.0996947007 \backslash \mathrm{Si},-1.6305692822,0,0.1$ Version=x86-Linux -G98RevA.11.3 \St at $=1-\mathrm{A} 1 \backslash \mathrm{HF}=-3184.6413761 \backslash \mathrm{RMSD}=1.375 \mathrm{e}-09 \backslash \mathrm{RMSF}=5.306 \mathrm{e}-0$ $5 \backslash \mathrm{ZPE}=9.5998896 \backslash \mathrm{Dipole}=-0.6263452,0 ., 0 . \backslash \mathrm{DipoleDeriv}=-0.2614116,0.03929$ $98,0.0141354,0.0656558,-0.1487302,-0.0283358,0.0064007,-0.0605111,-0.3$ $067468,-0.2614116,-0.0392998,0.0141354,-0.0656558,-0.1487302,0.0283358$ $, 0.0064007,0.0605111,-0.3067468,-0.2614116,-0.0392998,-0.0141354,-0.06$ $56558,-0.1487302,-0.0283358,-0.0064007,-0.0605111,-0.3067468,-0.261411$ $6,0.0392998,-0.0141354,0.0656558,-0.1487302,0.0283358,-0.0064007,0.060$ $5111,-0.3067468,-0.1073867,0 .,-0.0996224,-0.0000001,-0.2538528,0 .,-0.0$ $629402,0 ., 0.0083862,-0.1073867,0 ., 0.0996224,0.0000001,-0.2538528,0 ., 0$. $0629402,0.0 .0083862,-0.1697971,-0.0244883,0 .,-0.0245641,-0.2292301,0$. $, 0 ., 0 .,-0.1455885,-0.1697971,0.0244883,0 ., 0.0245641,-0.2292301,0 \ldots, 0 ., 0$ $., 0.1455885,-0.0398865,0.0 .0583176,0 .,-0.2563961,0 ., 0.2026114,0 .,-0$. $2032644,-0.0398865,0 .,-0.0583176,0 .,-0.2563961,0 .,-0.2026114,0 .,-0.203$ $2644,-0.3202133,0 ., 0 ., 0.0000002,0.0738783,0 ., 0 ., 0 .,-0.09208 \backslash$ Polar $=416$. $1319758,-0.0000007,446.4737941,-0.0000024,-0.0000033,493.9283693 \backslash \mathrm{PG}=\mathrm{C} 0$ 2V [C2 (Si1), SGV (Si4), SGV' (Si2),X(Si4)] \NImag=0 \\0.03478795,0.00623160, $0.06705153,-0.01033552,-0.00175940,0.06687648,-0.00188157,-0.00106858$,

$1 \backslash 1 \backslash$ FAU-CCC-AW433B \Freg \RB3LYP \6-311+G(d) \Si12 (2-) \SCHLEYER $24-N O V-2002 \backslash 0 \backslash \backslash \# B 3 I$ $\mathrm{YP} / 6-311+\mathrm{G} * \mathrm{FREQ}=$ NORAMAN GUESS=HUCKEL $\backslash \backslash \mathrm{CLOSO}-\mathrm{SI} 12-2 \mathrm{IH} \backslash \backslash-2,1 \backslash \mathrm{Si}, 0,0,2.4738375$ $232 \backslash \mathrm{Si}, 1.3005733523,-1.7900856483,1.1063337734 \backslash \mathrm{Si},-1.3005733523,-1.7900856483,1$ $.1063337734 \backslash \mathrm{Si},-2.1043718888,0.6837518749,1.1063337734 \backslash \mathrm{Si}, 0 ., 2.2126675469,1.106$ $3337734 \backslash \mathrm{Si}, 2.1043718888,0.6837518749,1.1063337734 \backslash \mathrm{Si}, 0 .,-2.2126675469,-1.106333$ $7734 \backslash \mathrm{Si},-2.1043718888,-0.6837518749,-1.1063337734 \backslash \mathrm{Si},-1.3005733523,1.7900856483$ $,-1.1063337734 \backslash \mathrm{Si}, 1.3005733523,1.7900856483,-1.1063337734 \backslash \mathrm{Si}, 2.1043718888,-0.68$ $37518749,-1.1063337734 \backslash \mathrm{Si}, 0 ., 0 .,-2.4738375232 \backslash \backslash$ Version=DEC-AXP-OSF / 1-G98RevA. 11 $.3 \backslash$ State $=1-A G \backslash H F=-3474.1430685 \backslash \mathrm{RMSD}=1.051 \mathrm{e}-09 \backslash \mathrm{RMSF}=3.839 \mathrm{e}-05 \backslash \mathrm{ZPE}=10.0538654 \backslash \mathrm{Dip}$ ole $=0 ., 0 ., 0 . \backslash$ DipoleDeriv $=-0.2448879,0 ., 0 ., 0 .,-0.2449128,-0.0000301,0 .,-0.000105$ Polar $=466.6378895,0 ., 466.5571406,0 .,-0.0242212,466.6015786 \backslash \mathrm{PG}=\mathrm{IH} \quad[6 \mathrm{C} 5(\mathrm{Si} 1 . \mathrm{Si} 1)]$ $\backslash \mathrm{NImag}=0 \backslash \backslash 0.03895884,0 ., 0.03895844,0 ., 0.00000011,0.07450254,-0.00744021,0.00523$ $\backslash 0 ., 0 .,-0.00006650,-0.00003496,0.00004812,-0.00002974,0.00003496,0.00004812,-0$. $\backslash \backslash \dot{0}$

\section{MO-NICS analysis}

\section{MAG-ReSpect copyright information:}


version 1.0

Developed by :

Vladimir G. Malkin

Olga L. Malkina

Roman Reviakine

Bernd Schimmelpfennig

Martin Kaupp

using parts of the deMon code

and integrals from the HERMIT code [2]

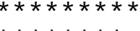

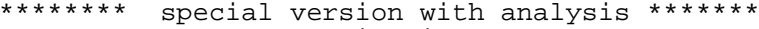

$\star \star \star \star *$ of separate contributions to g-tensor) $\star \star \star *$

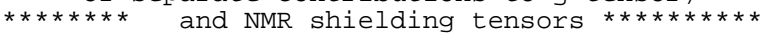

$\star \star \star * *$ A.Arbuznikov - A.Patrakov, March $2002 * * *$

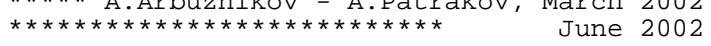




\section{MO analysis of NICS from MAG-ResPect output}

Total shieldings differ slightly from values of Table 1 due to the different numerical implementations of the GIAO method in Gaussian and MAG-Respect.

$\mathrm{B}_{6} \mathrm{H}_{6}{ }^{2-}$

NMR SHIELDING TENSOR FOR $X X$

$$
\text { at } \mathrm{X}=0.000000 \quad \mathrm{Y}=
$$

$0.000000 \begin{array}{cc}(\text { NUCLEUS NUMBER }= & 1) \\ Z= & 0.000000^{1}\end{array}$

NMR SHIELDING CONSTANT

29.1

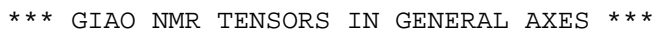

$\begin{array}{lrrrrrrrrr}\text { D } & -8.0 & 0.0 & 0.0 & 0.0 & -8.0 & 0.0 & 0.0 & 0.0 & -8.0 \\ \text { P0 } & 120.5 & 0.0 & 0.0 & 0.0 & 120.5 & 0.0 & 0.0 & 0.0 & 120.5 \\ \text { P1 } & -83.4 & 0.0 & 0.0 & 0.0 & -83.4 & 0.0 & 0.0 & 0.0 & -83.4 \\ \text { SUM } & 29.1 & 0.0 & 0.0 & 0.0 & 29.2 & 0.0 & 0.0 & 0.0 & 29.1\end{array}$

*** GIAO NMR TENSORS IN PRINCIPAL AXES ***

\begin{tabular}{lrrr|rrrr} 
Sigma-d (i,i) & -8.0 & -8.0 & -8.0 & \multicolumn{4}{c}{ PRINCIPAL AXES } \\
Sigma-p0 (i,i) & 120.5 & 120.5 & 120.5 & 1 AXIS & 0.5123 & 0.3660 & 0.7769 \\
Sigma-p1 $(i, i)$ & -83.4 & -83.4 & -83.3 & 2 AXIS & $-0.6205-0.4676$ & 0.6296 \\
SIGMA (i,i) & 29.1 & 29.1 & 29.2 & 3 AXIS & $0.5937-0.8046$ & -0.0124
\end{tabular}

*** MO CONTRIBUTIONS TO SIGMA IN PRINCIPAL AXES *** corresponding eigen values:

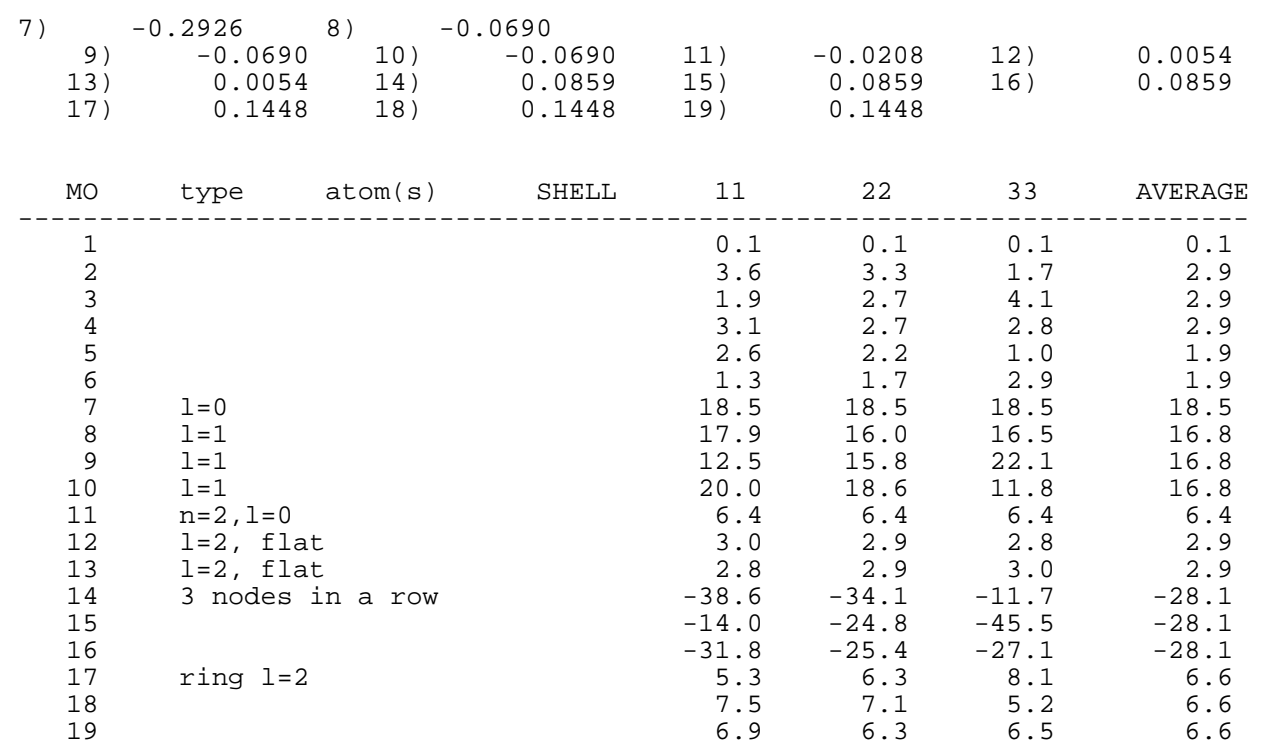


$\mathbf{S i}_{6}$

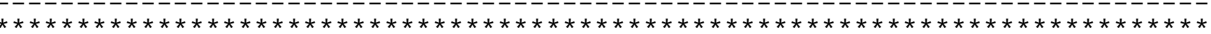

NMR SHIELDING TENSOR FOR XX

(NUCLEUS NUMBER $=1$ )

at $\mathrm{X}=0.000000 \quad \mathrm{Y}=$

0.000000

$\mathrm{Z}=$

0.000000

NMR SHIELDING CONSTANT $=-15.7$

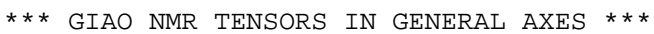

$\begin{array}{lrrrrrrrrr}\text { D } & -34.0 & 0.0 & 0.0 & 0.0 & -34.0 & 0.0 & 0.0 & 0.0 & -34.0 \\ \text { P0 } & 145.4 & 0.0 & 0.0 & 0.0 & 145.4 & 0.0 & 0.0 & 0.0 & 145.4 \\ \text { P1 } & -127.1 & -0.1 & 0.0 & 0.0 & -127.2 & 0.0 & 0.0 & 0.0 & -127.1 \\ \text { SUM } & -15.7 & -0.1 & 0.0 & 0.0 & -15.7 & 0.0 & 0.0 & 0.0 & -15.7\end{array}$

* GIAO NMR TENSORS IN PRINCIPAL AXES ***

\begin{tabular}{lr|rrr|rrr} 
Sigma-d $(i, i)$ & -34.0 & -34.0 & -34.0 & \multicolumn{3}{c}{ PRINCIPAL AXES } \\
Sigma-p0 $(i, i)$ & 145.4 & 145.4 & 145.4 & 1 AXIS & 0.6074 & 0.7920 & -0.0614 \\
Sigma-p1 $(i, i)$ & -127.2 & -127.1 & -127.1 & 2 AXIS & $0.1505-0.0389$ & 0.9878 \\
SIGMA (i,i) & -15.8 & -15.7 & -15.7 & 3 AXIS & $0.7800-0.6092-0.1428$
\end{tabular}

*** MO CONTRIBUTIONS TO SIGMA IN PRINCIPAL AXES ***

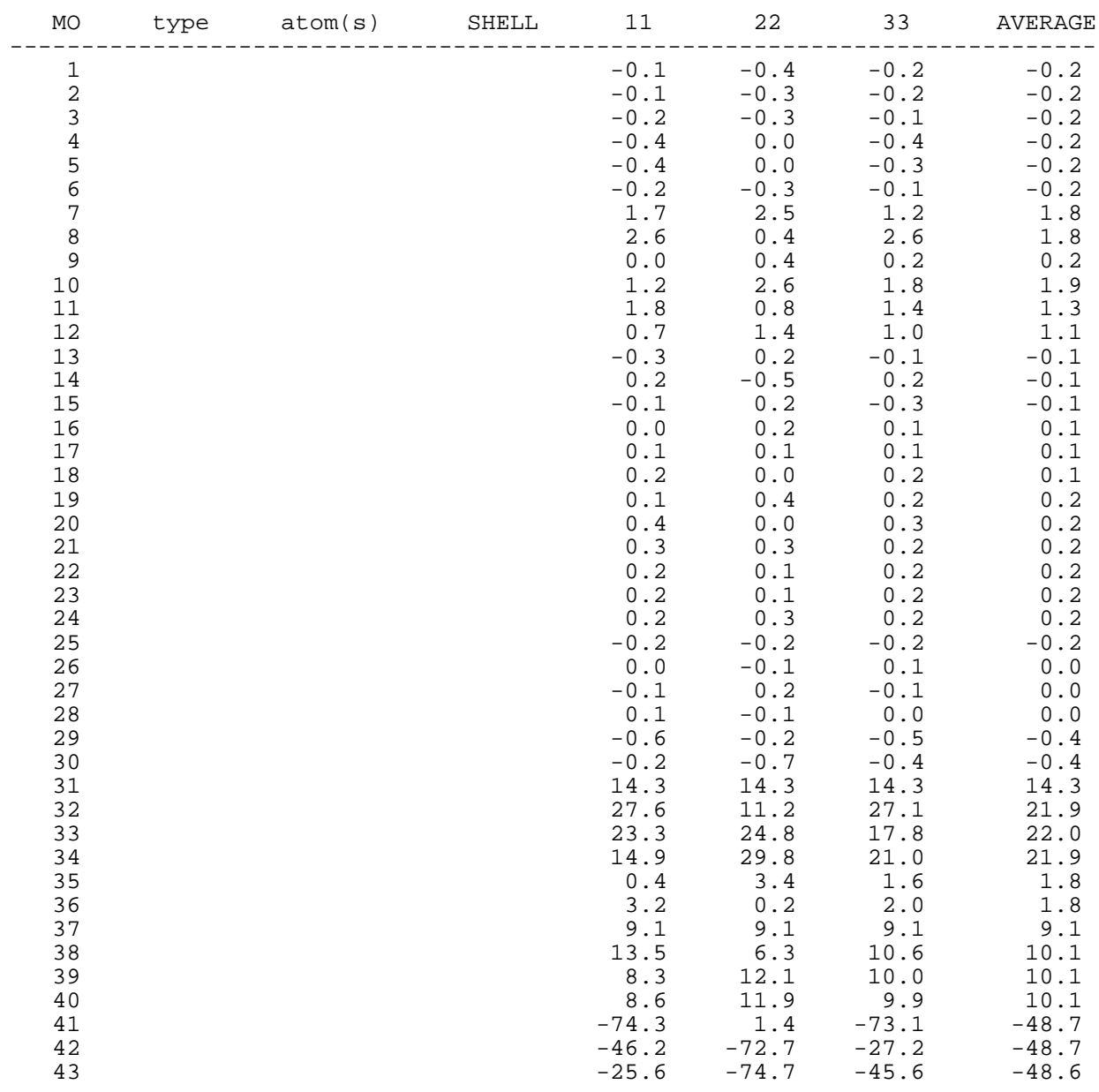


$\mathrm{B}_{12} \mathrm{H}_{12}{ }^{2}$

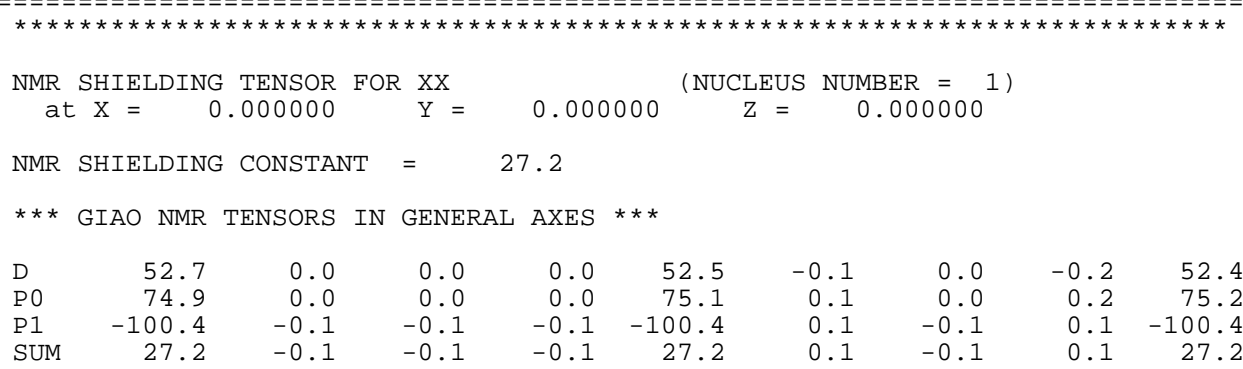

*** GIAO NMR TENSORS IN PRINCIPAL AXES ***

\begin{tabular}{lr|rrr|rrrr} 
Sigma-d $(i, i)$ & 52.6 & 52.6 & 52.4 & & \multicolumn{2}{c}{ PRINCIPAL AXES } \\
Sigma-p0 $(i, i)$ & 75.0 & 75.0 & 75.2 & 1 AXIS & 0.5321 & 0.8214 & -0.2054 \\
Sigma-p1 $(i, i)$ & -100.5 & -100.4 & -100.2 & 2 AXIS & 0.6101 & -0.2037 & 0.7657 \\
SIGMA & $(i, i)$ & 27.1 & 27.1 & 27.3 & 3 AXIS & -0.5871 & 0.5327 & 0.6095 \\
\end{tabular}

*** MO CONTRIBUTIONS TO SIGMA IN PRINCIPAL AXES ***

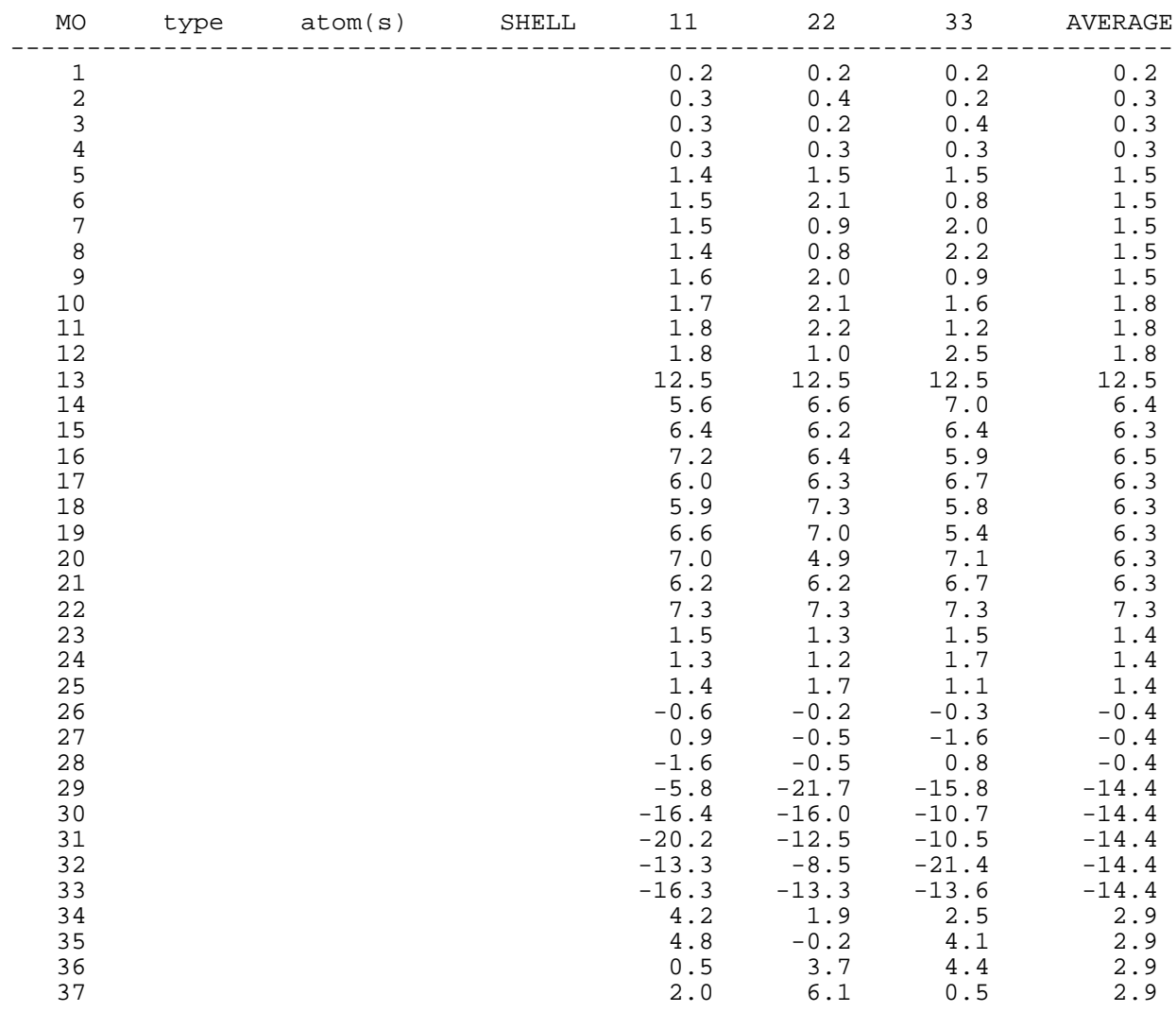


Si

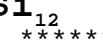

NMR SHIELDING TENSOR FOR XX

at $\mathrm{X}=0.000000$

0.000000

$($ NUCLEUS NUMBER $=1$ )

NMR SHIELDING CONSTANT

$-51.6$

*** GIAO NMR TENSORS IN GENERAL AXES ***

$\begin{array}{lrrrrrrrrr}\text { D } & 29.1 & 0.0 & 0.0 & 0.0 & 29.1 & 0.0 & 0.0 & 0.0 & 29.1 \\ \text { P0 } & 57.9 & 0.0 & 0.0 & 0.0 & 57.9 & 0.0 & 0.0 & 0.0 & 57.9 \\ \text { P1 } & -138.6 & 0.0 & 0.0 & 0.0 & -138.6 & 0.1 & 0.0 & 0.1 & -138.5 \\ \text { SUM } & -51.6 & 0.0 & 0.0 & 0.0 & -51.6 & 0.1 & 0.0 & 0.1 & -51.5\end{array}$

* GIAO NMR TENSORS IN PRINCIPAL AXES ***

\begin{tabular}{lr|rr|rrrr} 
Sigma-d (i,i) & 29.1 & 29.1 & 29.1 & \multicolumn{4}{|c}{ PRINCIPAL AXES } \\
Sigma-p0 (i,i) & 57.9 & 57.9 & 57.9 & 1 AXIS & -0.2439 & -0.9347 & 0.2587 \\
Sigma-p1(i,i) & -138.7 & -138.6 & -138.4 & 2 AXIS & 0.9343 & -0.1549 & 0.3212 \\
SIGMA $(i, i)$ & -51.7 & -51.6 & -51.4 & 3 AXIS & -0.2601 & 0.3200 & 0.9110
\end{tabular}

*** MO CONTRIBUTIONS TO SIGMA IN PRINCIPAL AXES ***

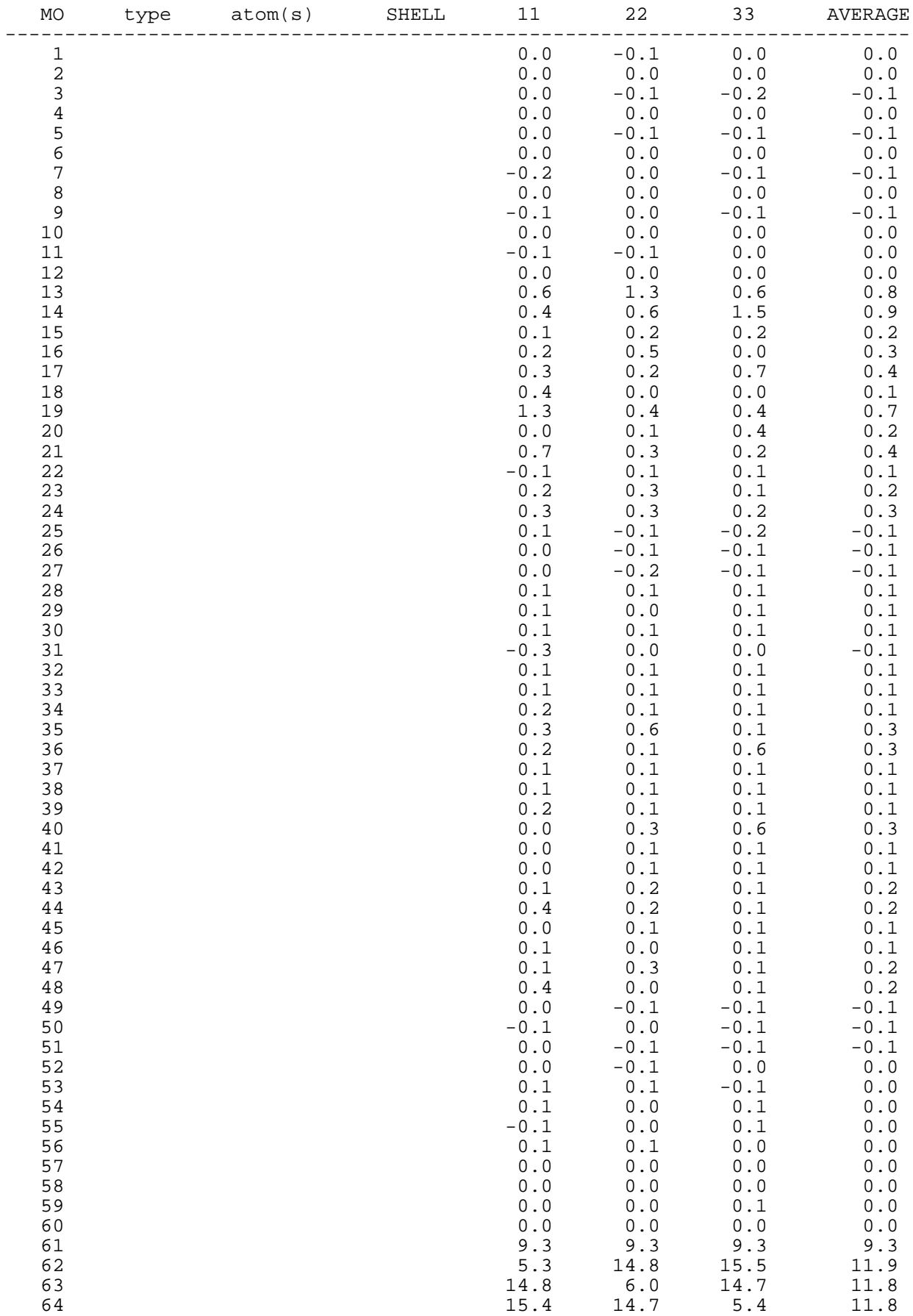




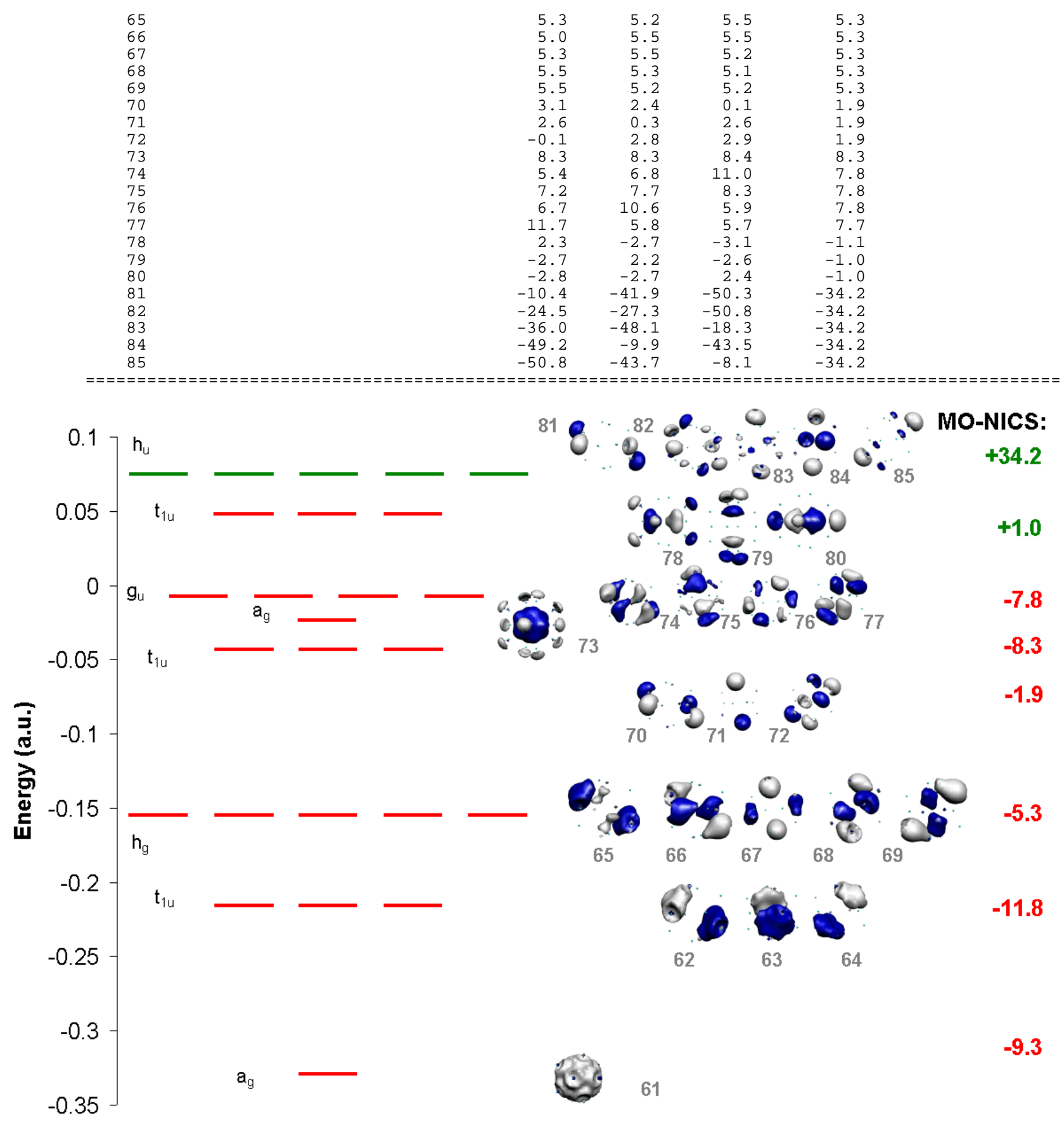




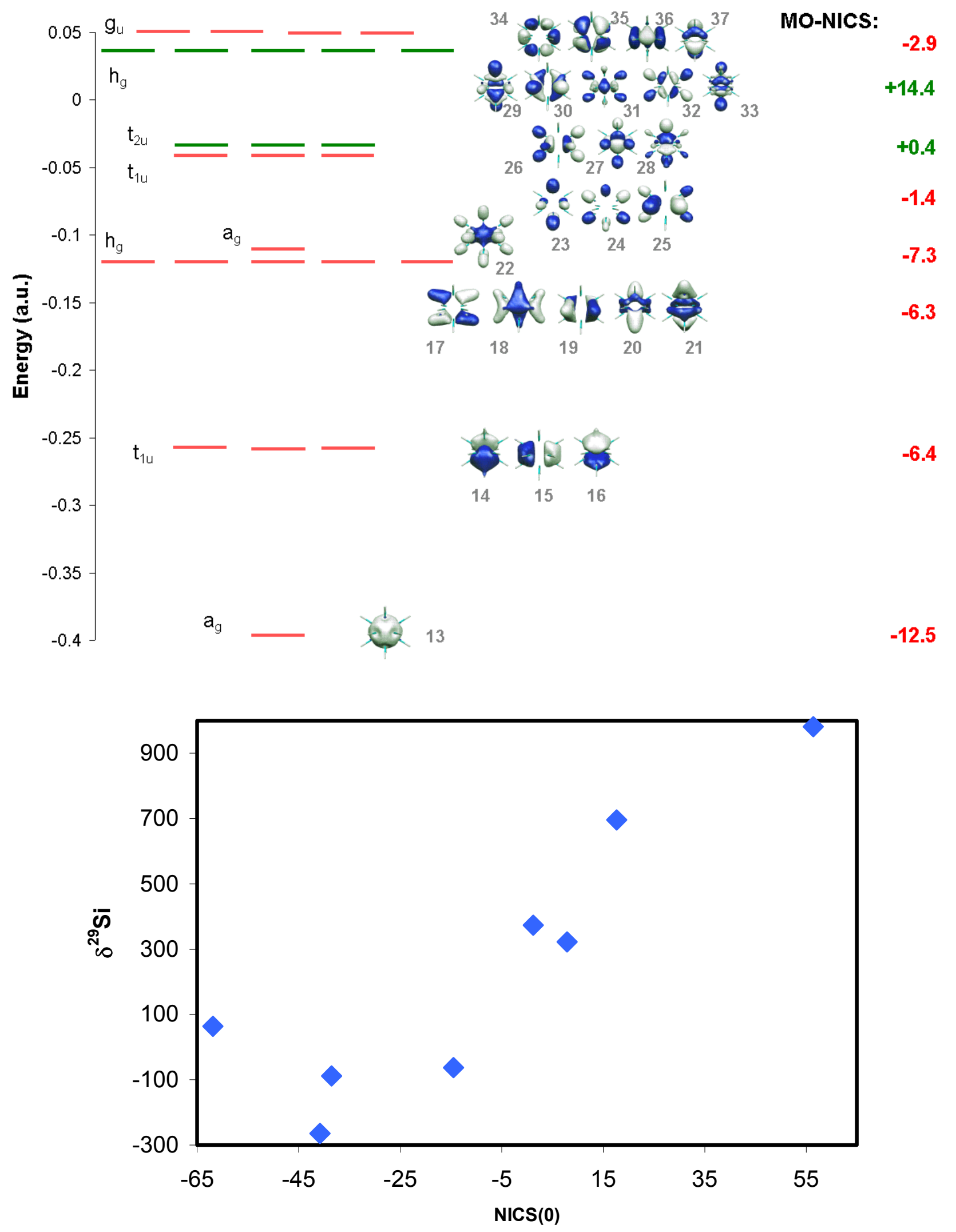

Figure S1: Scatter plot of the averaged ${ }^{29} \mathrm{Si}$ chemical shift vs. NICS (0), computed at the molecular centre, in ppm. 


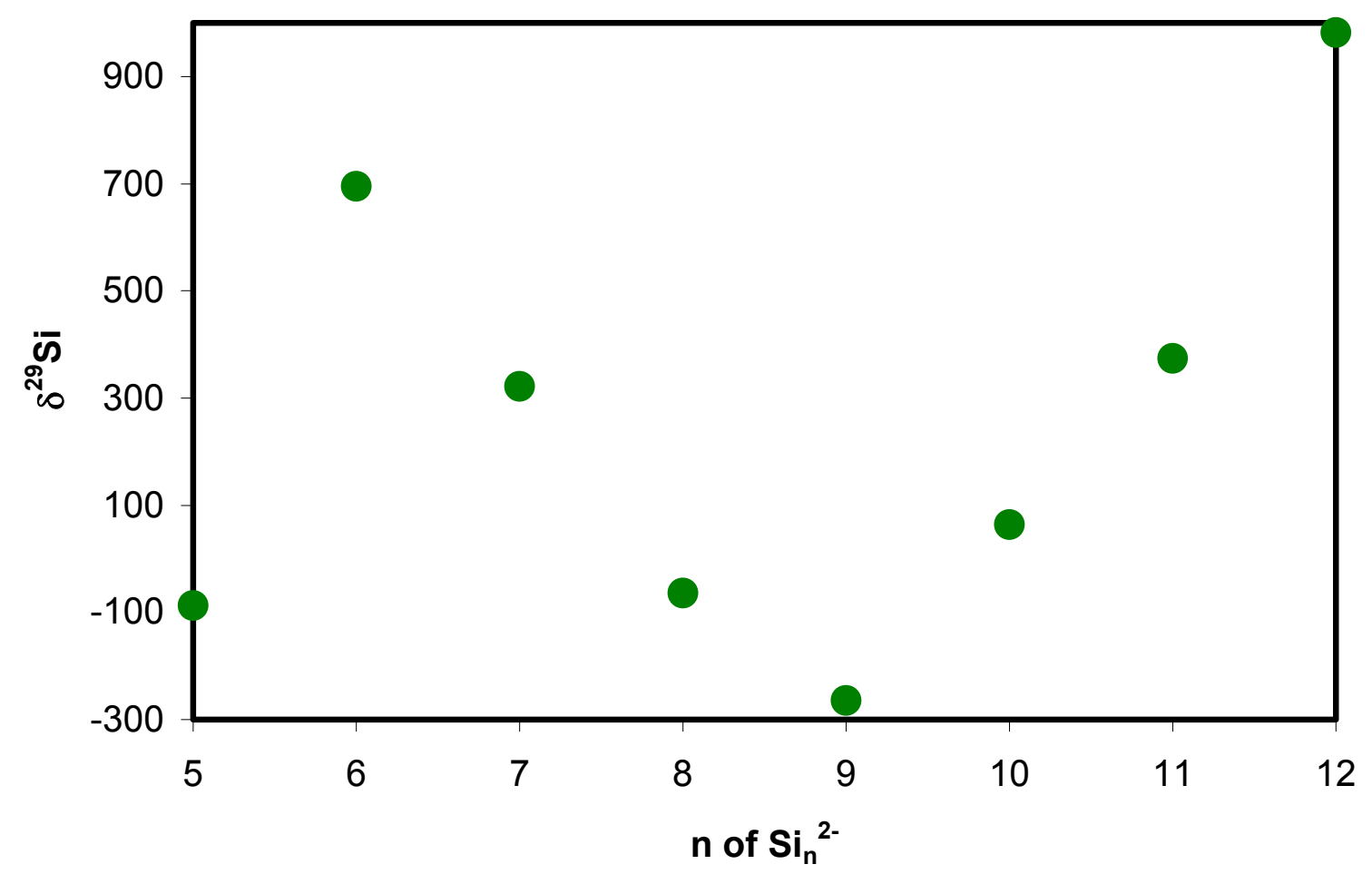

Figure $\mathrm{S} 2$ : Averaged ${ }^{29} \mathrm{Si}$ chemical shift of $\mathrm{Si}_{\mathrm{n}}{ }^{2-}$ clusters vs. vertex number $\mathrm{n}$.

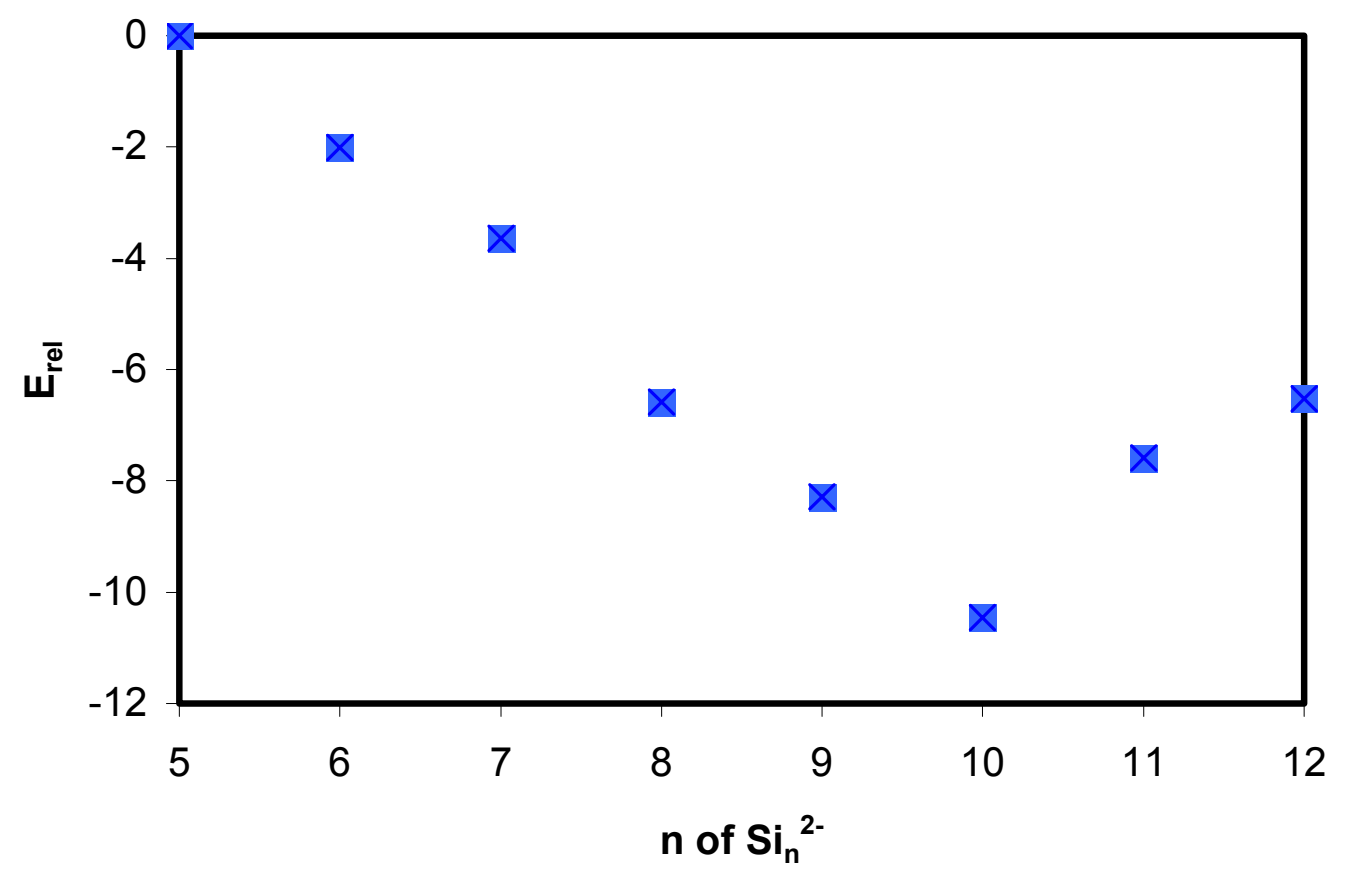

Figure S3: Relative energy per vertex with respect to $\mathrm{Si}_{5}{ }^{2-}$

$\mathrm{E}_{\mathrm{rel}}=\mathrm{E}_{\mathrm{tot}}\left(\mathrm{Si}_{\mathrm{n}}{ }^{2-}\right) / \mathrm{n}-\mathrm{E}_{\mathrm{tot}}\left(\mathrm{Si}_{5}{ }^{2-}\right) / 5$ in $\mathrm{kcal} /$ mole vs. vertex number $\mathrm{n}$ 


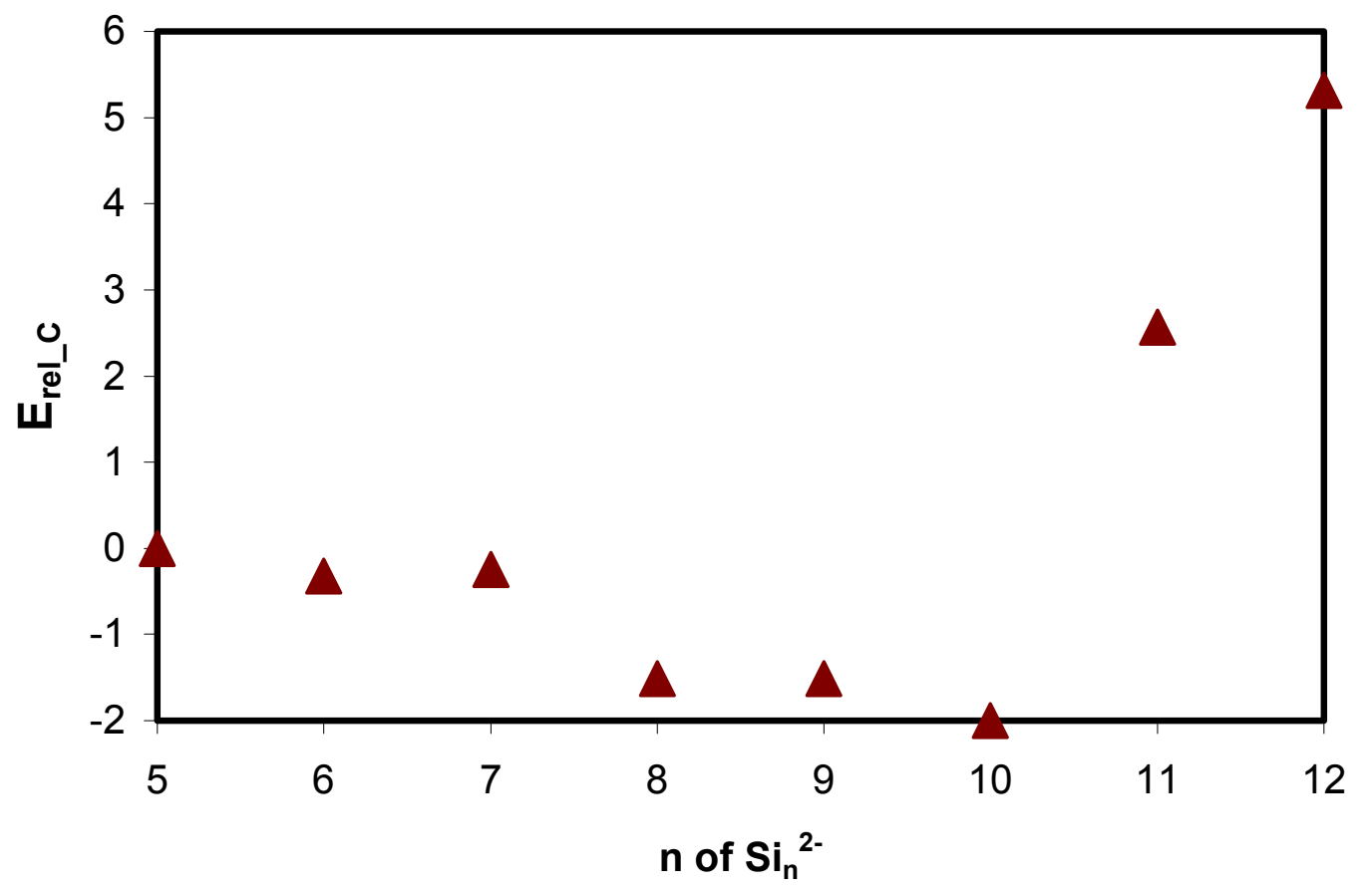

Figure S4: $\mathrm{E}_{\text {rel }}{ }^{\mathrm{C}}$ Relative energy corrected for the Coulomb repulsion $\mathrm{E}_{\text {rel }}{ }^{\mathrm{C}}=$ $\left.\left(E_{\text {tot }}(n) / n-Q / R_{n}\right)-\left(E_{\text {tot }}(5) / 5-Q / R_{5}\right)\right], Q=2, R$ is the distance of a vertex from the centre (in Bohr), transferred to $\mathrm{kcal} / \mathrm{mol}$, vs. vertex number $\mathrm{n}$ of $\mathrm{Si}_{\mathrm{n}}{ }^{2-}$ clusters. 